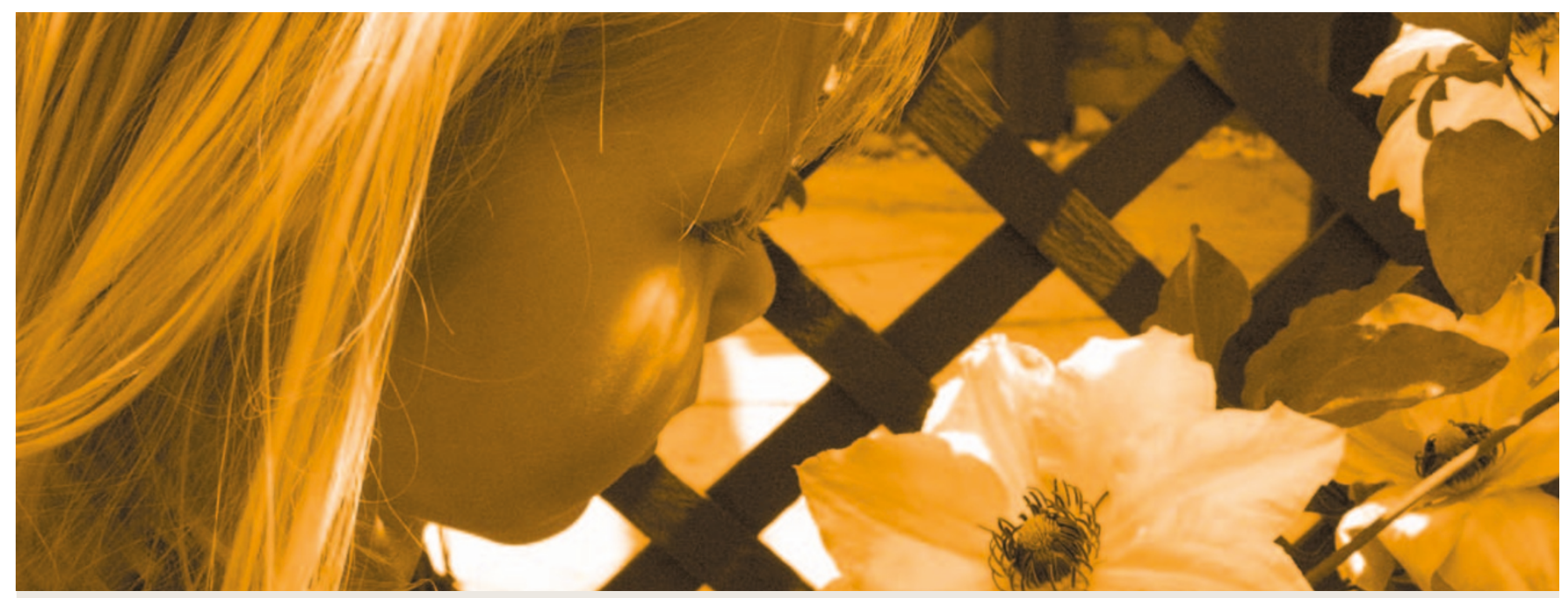

GENE REGULATION

\title{
Sniffing out regulation at a distance
}

Mammals have hundreds of genes that encode different olfactory receptors, with only one type of receptor being expressed in individual sensory neurons. This allows a discriminating sense of smell, but also poses a puzzle in terms of gene regulation: how do cells pick just one receptor gene to express from this large set of choices? Recent work from Richard Axel and colleagues provides an answer to this mystery, showing that long-range interactions within the genome hold the key.

One model for how a single odorant receptor (OR) is picked for expression involves a putative enhancer element that can activate all OR genes, but interacts with only one of them - which is chosen at random - in each cell. A candidate enhancer for this model is the $\mathrm{H}$ enhancer, which is a cis-regulatory element for some OR genes. To test whether this enhancer also interacts in trans to activate other genes of this class, the authors used the chromosome conformation capture (3C) technique, which involves crosslinking chromatin and using PCR to identify the interacting DNA sequences. When applied to chromatin from mouse olfactory neurons, this showed that the $\mathrm{H}$ enhancer does indeed contact OR genes located on several other chromosomes.

If the $\mathrm{H}$ enhancer is a trans-acting activator for OR genes, it should only contact a particular OR gene promoter in cells that express the corresponding encoded receptor. Axel and colleagues confirmed that this is the case using fluorescence in situ hybridization (FISH) with probes specific for the $\mathrm{H}$ enhancer and OR promoters. By carrying out a combination of DNA FISH for the $\mathrm{H}$ enhancer and RNA FISH for specific transcripts, they also showed that the enhancer interacts specifically with the actively transcribed OR allele.

\section{This study [reveals] how the stochastic choice of a single receptor gene from a wide array of candidates can be achieved in the mammalian olfactory system.}

How is the expression of a single receptor gene ensured when two allelic copies of the $\mathrm{H}$ enhancer are present in the genome? The authors showed that a subset of $\mathrm{H}$ alleles is methylated at $\mathrm{CpA}$ dinucleotides in olfactory neurons only. Although the functional effects of this type of modification are as yet unclear, this methylation might provide a means by which these neurons inactivate one $\mathrm{H}$ allele per cell. An alternative model, supported by previous studies, is that once an OR gene is activated by one $\mathrm{H}$ allele, a negativefeedback mechanism prevents the second allele from activating the expression of a second OR. It is also possible that both mechanisms operate to ensure strict regulation of OR expression.

To test their model of how the expression of single ORs is achieved, the authors looked at the effect of introducing extra copies of the $\mathrm{H}$ enhancer. To avoid complications with negative feedback caused by the initial expression of one OR, the activation of OR pseudogenes - which have lost their protein-coding ability - was assessed. Just as predicted, in mice carrying extra transgenic copies of $\mathrm{H}$ the authors saw co-expression of an OR gene and an OR pseudogene, confirming the importance of a single $\mathrm{H}$ allele activating a single OR gene.

This study has answered a long-standing riddle, explaining how the stochastic choice of a single receptor gene from a wide array of candidates can be achieved in the mammalian olfactory system. It also provides another striking example of the importance of interchromosomal interactions, which is emerging as an important theme in the regulation of eukaryotic gene expression.

Louisa Flintoft

ORIGINAL RESEARCH PAPER Lomvardas, S. et al.

Interchromosomal interactions and olfactory receptor choice. Cell 126, 403-413 (2006)

FURTHER READING Mombaerts, P. Genes and ligands for odorant, vomeronasal and taste receptors. Nature Rev. Neurosci. 5, 263-278 (2004)

WEB SITE

Richard Axel's web site: http://www.cumc.columbia.edu/ dept/neurobeh/axel 